SMASIS2014-7645

\title{
GUIDED WAVE DELAMINATION DETECTION AND QUANTIFICATION WITH WAVEFIELD DATA ANALYSIS
}

\author{
Zhenhua Tian \\ Department of Mechanical Engineering, \\ University of South Carolina, \\ Columbia, SC 29208, tianz@email.sc.edu \\ Jeffrey P. Seebo \\ Analytical Mechanics Associates, Inc. \\ Hampton, VA 23681, jeffrey.p.seebo@nasa.gov
}

\author{
Cara A.C. Leckey \\ Nondestructive Evaluation Sciences Branch, \\ NASA Langley Research Center, \\ Hampton, VA 23681, cara.ac.leckey@nasa.gov

\section{Lingyu Yu} \\ Department of Mechanical Engineering, \\ University of South Carolina, \\ Columbia, SC 29208, yu3@cec.sc.edu
}

\begin{abstract}
Unexpected damage can occur in aerospace composites due to impact events or material stress during off-nominal loading events. In particular, laminated composites are susceptible to delamination damage due to weak transverse tensile and interlaminar shear strengths. Developments of reliable and quantitative techniques to detect delamination damage in laminated composites are imperative for safe and functional optimally-designed next-generation composite structures. In this paper, we investigate guided wave interactions with delamination damage and develop quantification algorithms by using wavefield data analysis. The trapped guided waves in the delamination region are observed from the wavefield data and further quantitatively interpreted by using different wavenumber analysis methods. The frequency-wavenumber representation of the wavefield shows that new wavenumbers are present and correlate to trapped waves in the damage region. These new wavenumbers are used to detect and quantify the delamination damage through the wavenumber analysis, which can show how the wavenumber changes as a function of wave propagation distance. The location and spatial duration of the new wavenumbers can be identified, providing a useful means not only for detecting the presence of delamination damage but also allowing for estimation of the delamination size. Our method has been applied to detect and quantify real delamination damage with complex geometry (grown using a quasi-static indentation technique). The detection and quantification results show the location, size, and shape of the delamination damage.
\end{abstract}

\section{INTRODUCTION}

Aviation safety is an area of focus in NASA's aeronautics work. Damage due to impact events must be detected and the damage must be evaluated to determine if repairs are needed for safe operation. Matrix cracking, fiber breakage, fibermatrix debonding, and delamination are the most common types of damage in fiber composites [1]. In particular, laminated composites are susceptible to delamination damage due to their weak transverse tensile and interlaminar shear strengths (as compared to their in-plane properties) [1]. Delamination damage can occur largely internally to the composite such that a damage indication is barely visible on the composite surface to the naked eye; such damage is known as Barely Visible Impact Damage (BVID) [2]. Damage below the BVID limit is not visible on the composite surface, even if delamination damage exists internally. Such hidden delamination damage must be detected and evaluated before it becomes critical. The problem of how best to detect such material changes in laminated composite components is still being solved, and a solution to this problem will be imperative for safe and functional, optimally-designed next-generation composite aircraft. Current composite "safety margin" design comes with a weight penalty which could be mitigated by the development of accurate, efficient, and reliable structural health monitoring (SHM) methods to locate and quantify the unique damage types that can occur in composite materials.

Guided ultrasonic waves have proven effective for SHM due to their ability to inspect a large area while maintaining the sensitivity to small defects in the structure [3-8]. Advances in guided wave based SHM technologies over the last decade have 
demonstrated the feasibility of detecting and locating damages in composite structural components [9-17]. Due to the complex wave interaction that occurs when hidden delamination damage is present, tremendous efforts have been put into delamination detection methodologies and the study of wave interaction with this damage type, such as references [17-23].

In this paper, we study guided wave interactions with the delamination damage and apply wavefield analysis methodologies to analyze the complex wave interaction phenomenon and to quantify the delamination damage. A scanning laser Doppler vibrometer is used to measure the timespace wavefield of guided wave interactions with the delamination. Three dimensional (3D) Fourier transform (FT) was used to transform the wavefield data from the time-space domain into frequency-wavenumber domain where further wave propagation information is revealed. To detect and quantify the delamination damage, we apply a wavenumber field analysis method which can provide the wavenumber value at each spatial location. The wavenumber field analysis method is then applied to inspect a plate with impact induced delamination damage. The detection and quantification results show clear indication of the location, size, and shape of the delamination damage.

\section{GUIDED WAVES IN LAMINATED COMPOSITES}

When guided waves propagate in a delaminated composite, multiple reflections within the delamination region can occur, as shown in references [24-28]. Hence, a considerable amount of ultrasonic energy is "trapped" above and below delaminated regions until eventually the energy dissipates. Ramadas et al. studied the interaction of the antisymmetric $\mathrm{A}_{0}$ mode with symmetric delaminations [25]. They also identified multiple reflections in the delamination region. The trapped energy phenomenon of guided wave propagation in delaminated composites has also been studied experimentally through the use of wavefield images obtained from laser vibrometry tests [26, 27]. It is observed that upon entering the delaminated region, the incident waves will be split above and below the delamination and then propagate independently through upper and lower lamina (single delamination scenario being assumed). After that, a significant portion of the waves are reflected back from the far edge of the delamination (exit region). The reflected waves then pass back above (or below) the delaminated area and experience reflections at the original entrance to the delamination region. The process is repeated until the energy is dissipated, as found in the simulation work reported by Hayashi and Kawashima[24]. Glushkov et al. also observed similar wave energy trapping and localization in a strip delamination by using laser vibrometry to record the wavefield [28].

Since the material layers above and below the delamination have different thicknesses from the original plate thickness, the trapped waves will accordingly have different wave propagation characteristics. Therefore, it is expected that by analyzing the trapped wave signals, the delamination dimensions can be quantified. Prior work by Rogge and Leckey demonstrated that wavenumber analysis can be used to determine the approximate depth and size of near-surface delamination damage [23]. This paper demonstrates quantitative characterization of the trapped waves, along with delamination detection and sizing utilizing the trapped wave phenomenon for real delamination damage.

To demonstrate the fundamentals of guided wave propagation and interactions in laminated composites, two 380 $\mathrm{mm} \times 380 \mathrm{~mm}$ 8-ply IM7/8552 composite laminate plates with layup $\left[\mathrm{O}_{2} / 90_{2}\right]_{\mathrm{s}}$ are first studied. One plate was pristine, while the other contained a delamination created by inserting a $20 \mathrm{~mm} \times 20 \mathrm{~mm}$ Teflon film under the second ply during manufacturing. Guided waves are actuated by a surface bonded $7 \mathrm{~mm}$ round PZT patch at $300 \mathrm{kHz}$, while the timespace wavefield data are acquired by a scanning laser Doppler vibrometer and are used to investigate the wave propagation [29]. Figure 1 shows the schematic of the test plate (detailed experimental setup can be found in [29]).

Figure 2 shows the time-space wavefields of the guided waves propagating along the $y$-axis (1D wave propagation). For the pristine case in Figure 2a, a faster $\mathrm{S}_{0}$ mode and a slower $\mathrm{A}_{0}$ mode can clearly be observed. For the delaminated case in Figure $2 b$, waves propagating back and forth between the beginning $(y=20 \mathrm{~mm})$ and ending $(\mathrm{y}=40 \mathrm{~mm})$ boundaries of the delamination can be distinctively seen, exhibiting a "trapped wave" phenomenon [27].

Figure 3 shows the snapshots of the wavefields in the scan area. Figure $3 \mathrm{a}$ is the wave propagation recorded at $15 \mu$ s when the incident $\mathrm{S}_{0}$ mode interacts with the delamination damage. We see there are waves propagating both upward and downward between delamination boundaries (upper and lower). At $30 \mu \mathrm{s}$, as shown in Figure $3 \mathrm{~b}$, the incident $\mathrm{A}_{0}$ mode is interacting with the delamination damage. The figure shows a localized yet complicated interference pattern in the delamination region due to the multiple trapped waves and wave interferences at this time.

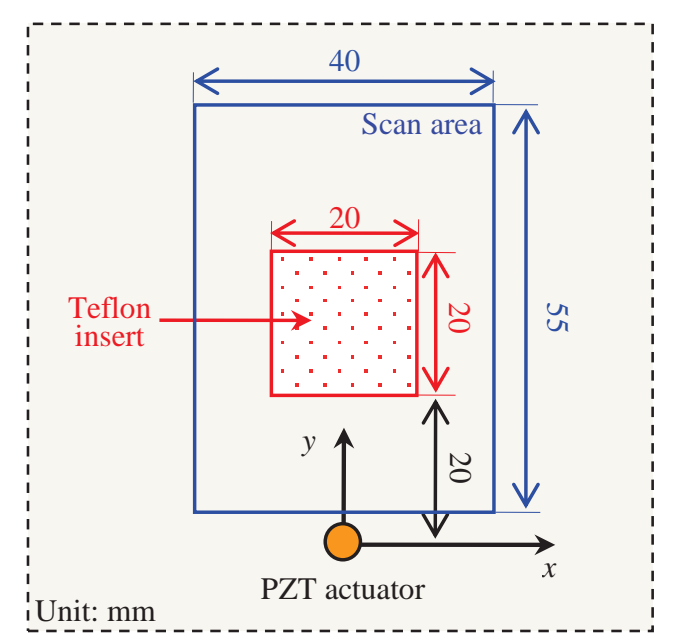

Figure 1. Composite plate setup for the laser vibrometry test. 


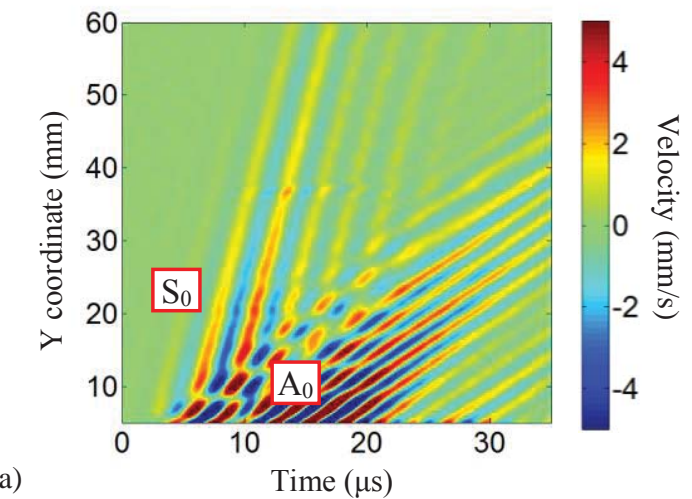

(a)

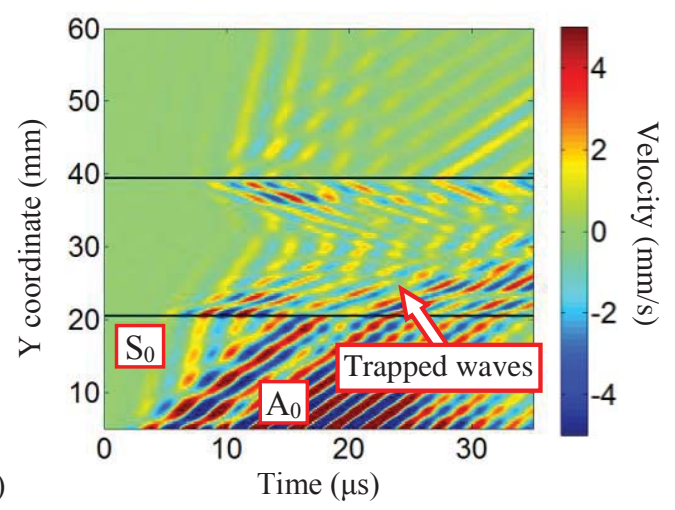

Figure 2. The time-space wavefields of the waves propagating along the y axis: (a) for the pristine plate, and (b) for the delaminated plate. The two solid black lines at $20 \mathrm{~mm}$ and $40 \mathrm{~mm}$ are the delamination boundaries.

\section{DATA ANALYSIS APPROACHES}

Though interesting wave interactions have been observed in the time-space wavefield, a quantitative evaluation is not yet readily obtained. To quantify the complicated wave interactions within the delamination region, further wave data analysis becomes essential. Wave propagation characteristics such as wave mode content, as well as how the waves evolve as with frequency or space are also desired. Prior works reported in the literatures have demonstrated that wavefield data in the frequency-wavenumber representation have abundant information regarding the existence of various wave modes and wave propagation characteristics [21, 30-33]. We therefore develop and apply several quantitative wave analysis approaches based on wavenumber information in order to study wave interactions with delamination type defects, and to detect delamination damage quantitatively.

\subsection{Frequency-wavenumber spectral analysis}

Frequency-wavenumber information of the guided waves can be obtained from the time-space wavefield by applying a 3D FT, mathematically given as
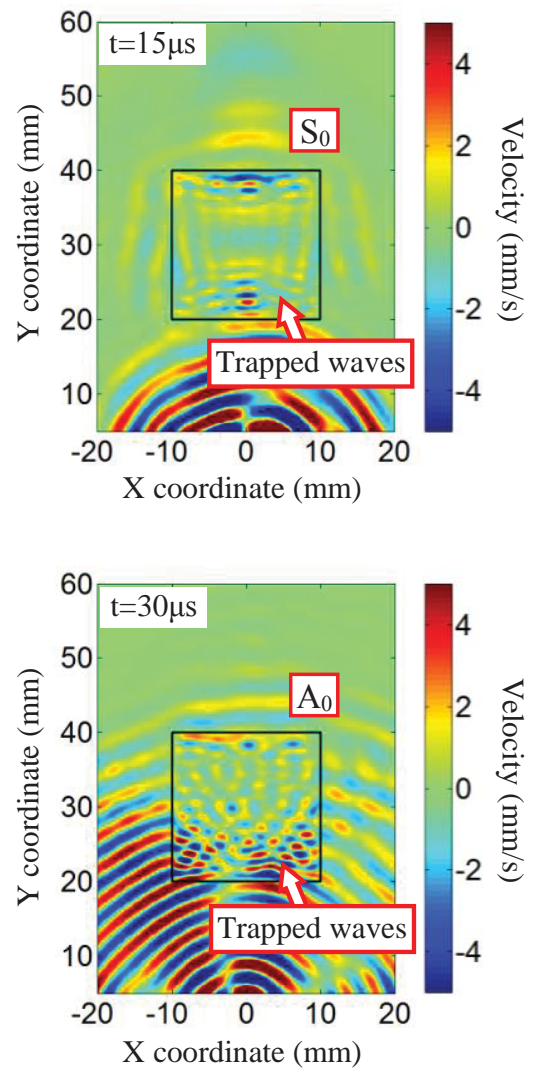

Figure 3. Guided wave propagation and "trapping" in the delaminated composite plate: (a) the $S_{0}$ mode interacting with the delamination at $15 \mu \mathrm{s}$, and (b) the $A_{0}$ mode interacting with the delamination at $30 \mu \mathrm{s}$. The black solid lines are the delamination boundaries.

$$
U(f, \mathbf{k})=\mathrm{F}[u(t, \mathbf{x})]=\int_{-\infty}^{\infty} \int_{-\infty}^{\infty} u(t, \mathbf{x}) e^{-j(2 \pi f t-\mathbf{k} \cdot \mathbf{x})} d t d \mathbf{x}
$$

where the space vector $\mathbf{x}$ and the wavenumber vector $\mathbf{k}$ in Cartesian coordinates are defined as $(x, y)$ and $\left(k_{x}, k_{y}\right) . u(t, \mathbf{x})$ is the time-space wavefield in terms of the time $t$ and the space vector $\mathbf{x} . U(f, \mathbf{k})$ is the resulting frequency-wavenumber representation or "spectrum" in terms of the frequency $f$ and the wavenumber vector $\mathbf{k}$. As the frequency is the counter part of time, the wavenumber is the counter part of space. The mathematics of the frequency-wavenumber technique can be found in several references [21, 30-34].

Figure $4 \mathrm{a}$ and $4 \mathrm{~b}$ show plots of the frequencywavenumber spectra of the experimental wavefields for the pristine and delaminated cases, respectively. To identify the wave mode information, the frequency-wavenumber spectra are compared with the theoretical dispersion curves which are plotted as dotted $\left(\mathrm{A}_{0}\right)$ and solid $\left(\mathrm{S}_{0}\right)$ lines. The pristine plate frequency-wavenumber spectrum (Figure $4 \mathrm{a}$ ) shows the lower amplitude $\mathrm{A}_{0}$ and higher amplitude $\mathrm{S}_{0}$ modes, which match well with the theoretical dispersion curves. 
In the delaminated plate, the delamination damage splits the originally bonded full laminate into two parts (above and below the delamination). Since the two separated parts have different layups and thinner thicknesses compared to the original pristine laminate, the guided waves in the separated parts will have modified propagation characteristics (such as modified dispersions and wavenumbers).

As expected, the spectrum of the delaminated case (Figure 4b) shows new frequency-wavenumber components in addition to the original $A_{0}$ and $S_{0}$ modes. The new frequencywavenumber components include both positive and negative counterparts, which correspond to the forward and backward propagating waves, respectively. We compared the new frequency-wavenumber components with the dispersion curves of the material above the delamination, equivalent to a two ply [0\0] laminate, since only the waves in the top plate surface was measured in the experiment. The comparison in Figure 4b shows the new frequency-wavenumber components match well with the theoretical curves of $A_{0}$ mode in the two ply [0\0] laminate. This analysis result agrees well with the real delamination depth which is below the second ply.

(a)

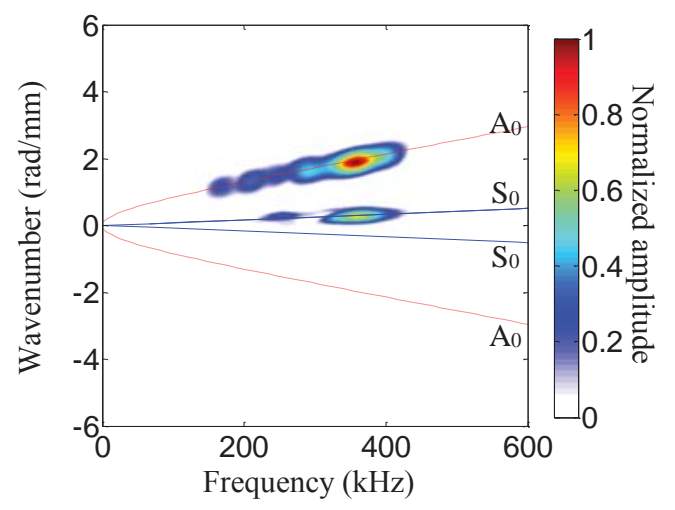

(b)

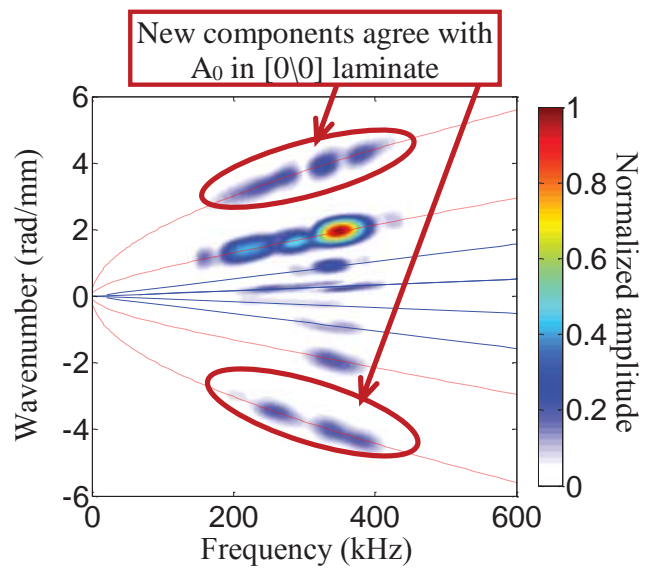

Figure 4. The frequency-wavenumber spectra of the timespace wavefields in Figure 2: (a) for the pristine plate, and (b) for the delaminated plate. The solid and dotted lines are theoretical dispersion curves for $S_{0}$ and $A_{0}$ wave modes, respectively.

\subsection{Spatial wave filtering}

The frequency-wavenumber analysis result shows that new frequency-wavenumber components appear in the spectrum of the delaminated plate, and are correlated to the waves in the delamination region. To further confirm this result, a wave filtering process is used to extract the new frequency-wavenumber components and transform them back into the time-space domain. The filtering process is mathematically expressed as the product between the frequency-wavenumber spectrum $U(f, \mathbf{k})$ and a filter function $F(f, \mathbf{k})$ as:

$$
U_{F}(f, \mathbf{k})=U(f, \mathbf{k}) F(f, \mathbf{k})
$$

where $F(f, \mathbf{k})$ denotes a filter in the frequency-wavenumber domain and $U_{F}(f, \mathbf{k})$ is the filtered spectrum, which only keeps the delamination induced new wavenumbers. By transforming the filtered spectrum $U_{F}(f, \mathbf{k})$ back into the time-space domain through inverse 3D FT, we can obtain the filtered time-space wavefield $u_{F}(t, \mathbf{x})$ as

$$
u_{F}(t, \mathbf{x})=\mathrm{F}^{-1}\left[U_{F}(f, \mathbf{k})\right]
$$

Since the filtered spectrum $U_{F}(f, \mathbf{k})$ only keeps the new frequency-wavenumber components, it is expected that the corresponding time-space wavefield $u_{F}(t, \mathbf{x})$ only contains the trapped waves induced by the delamination.

Figure 5 shows the filtered time-space wavefield along the y axis. Comparing this result to the wavefield before filtering (Figure 2b), it is seen that the filtered time-space wavefield shows trapped waves that exist between the delamination boundaries.

Figure 6 shows a snapshot at $30 \mu$ s of the filtered wavefield in the scan area. The filtered wavefield only contains the trapped waves in the delamination region, and shows the feasibility of using the trapped waves for delamination size quantification. Moreover, the wavefield filtering results further confirm that the new frequency-wavenumber components in Figure $4 \mathrm{~b}$ correspond to the trapped waves in the delamination region.

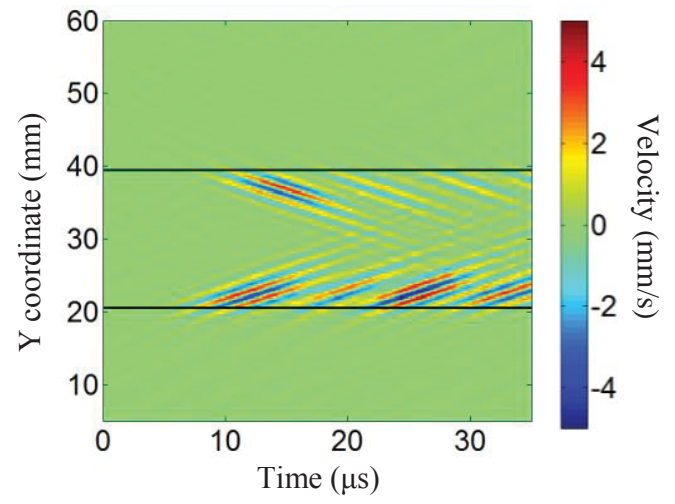

Figure 5. The filtered time-space wavefield of the waves propagating along y axis. The two solid black lines at $20 \mathrm{~mm}$ and $40 \mathrm{~mm}$ are the delamination boundaries. 


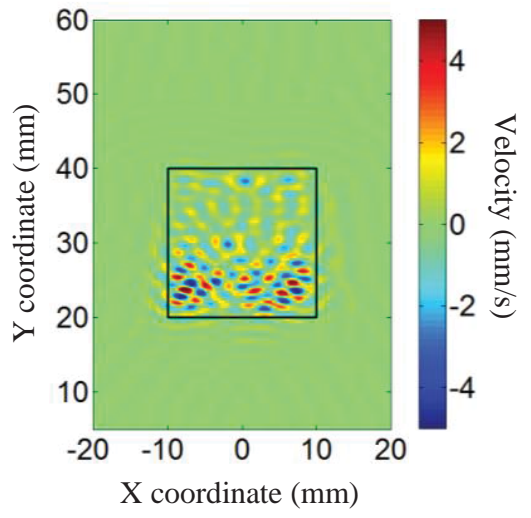

Figure 6. A snapshot at $30 \mu$ s of the filtered wavefield in the 2D scan area. The black solid lines are the delamination boundaries.

\subsection{Wavenumber field analysis}

The frequency-wavenumber analysis presented in the section 3.1 unveils wave propagation characteristics that cannot be explicitly seen in the time-space domain. However, in the resulting frequency-wavenumber representation $U(f, \mathbf{k})$, the spatial information $\mathbf{x}$ is not available from the result of the 3D FT (see Figure 4).

It is quite often desirable to know how the wavenumber changes in space. To retain the spatial information, a novel short space 3D FT has been applied to acquire a spacefrequency-wavenumber representation. The technique is a straightforward extension of the short time FT to multidimensional problems, i.e., breaking down the time-space wavefield into small segments over the space dimension before Fourier transformation [35]. To do this, the wavefield data is multiplied by a window function which is non-zero for only a short period in space while constant over the entire time dimension. The 3D FT is then applied to the resulting wavefield segments. When the window slides along the space dimension, a set of windowed wavefield segments is generated. The 3D FT is applied to these segments, resulting in a set of frequencywavenumber spectra that are indexed by the locations of the window. Through this technique the spatial information is retained. Mathematically, the short space 3D FT is implemented through spatial windowing technique, as

$$
S(\overline{\mathbf{X}}, f, \mathbf{k})=\int_{-\infty}^{\infty} \int_{-\infty}^{\infty} u(t, \mathbf{x}) W^{*}(t, \mathbf{x}-\overline{\mathbf{X}}) e^{-j(2 \pi t-\mathbf{k} \cdot \mathbf{x})} d t d \mathbf{x}
$$

where $\overline{\mathbf{X}}$ is the retained spatial vector and $W(t, \mathbf{x})$ is a window function. In our study, a Hanning function is used to construct the window $W(t, \mathbf{x})$, given as:

$$
W(t, \mathbf{x})=\left\{\begin{array}{cc}
0.5\left[1+\cos \left(2 \pi \frac{|\mathbf{x}|}{D_{x}}\right)\right] & \text { if }|\mathbf{x}| \leq D_{\mathbf{x}} / 2 \\
0 & \text { otherwise }
\end{array}\right.
$$

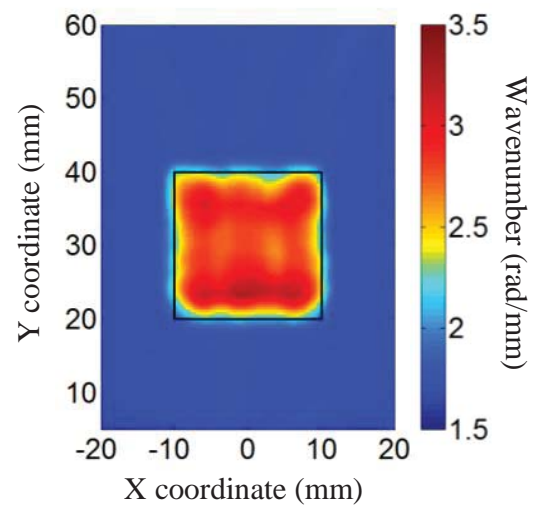

Figure 7. The calculated wavenumber field from the experimental time-space wavefield in the delaminated plate. The black solid lines are the delamination boundaries.

where $D_{\mathbf{x}}$ is the window length in the space domain. Further details of the short space 3D FT can be found in [23, 32, 33].

As stated above, by sliding the window $W(t, \mathbf{x})$ along the space dimension, the resulting space-frequency-wavenumber spectrum $S(\overline{\mathbf{X}}, f, \mathbf{k})$ can indicate how the frequency and/or wavenumber components vary in space. Thus, by finding the wavenumber vector $\mathbf{k}^{*}$ with the highest spectrum amplitude for each space and frequency point, we obtain the wavenumber vector function $\mathbf{k}^{*}(\overline{\mathbf{X}}, f)$, as

$$
\mathbf{k}^{*}(\overline{\mathbf{X}}, f)=\arg \max _{\mathbf{k}}|S(\overline{\mathbf{X}}, f, \mathbf{k})|
$$

Then, for a specific frequency $f_{0}$, we define the wavenumber field as

$$
k^{*}(\overline{\mathbf{X}})=\left|\mathbf{k}^{*}\left(\overline{\mathbf{X}}, f_{0}\right)\right|
$$

For a series of frequencies $f_{i}(i=1,2,3 \ldots N)$ within a small frequency band, the wavenumber field is defined as

$$
k^{*}(\overline{\mathbf{X}})=\frac{1}{N} \sum_{i=1}^{N}\left|\mathbf{k}^{*}\left(\overline{\mathbf{X}}, f_{i}\right)\right|
$$

The wavenumber field $k^{*}(\overline{\mathbf{X}})$ contains the local wavenumber information at each spatial point. Figure 7 gives the wavenumber field calculated from the time-space wavefield of the delaminated plate. The wavenumber field shows an area which has larger wavenumber values than the rest. This region matches well with the real delamination location and size. Since the delamination damage affects the wavenumber (as demonstrated in section 3.1), the wavenumber field can be used to detect and quantify the delamination location and size. 


\section{IMPACT-INDUCED DELAMINATION DETECTION AND QUANTIFICATION}

The previous section has shown that the wavenumber analysis approaches yields quantitative information of guided wave interactions and wave trapping in delamination regions; and can also be used as delamination detection tools. In this section, the wavenumber analysis methods are used to detect and quantify real impact-induced delamination damage in laminated composite plates.

\subsection{Damage growth in a CFRP plate}

A quasi-static indentation technique was used to grow damage in a CFRP plate. The technique has been shown by previous authors to be an ideal method for controlled growth of impact-like damage in composite laminates[36]. The composite sample is a 26 ply IM7/8552 $381 \mathrm{~mm} \times 381 \mathrm{~mm}$ laminate with a quasi-isotropic layup of $\left[\left(0_{2} / 45_{2} /-45_{2}\right)_{2} 90\right]_{s}$. The composite plate was loaded in a mechanical testing system using a 50.8 $\mathrm{mm}$ diameter indenter at a constant displacement rate of 1.27 $\mathrm{mm} / \mathrm{min}$. The displacement and load were monitored during the indentation test and the composite was unloaded when the data showed a sudden drop in load (due to damage growth), as shown in Figure 8b. A photo of the CFRP plate after the indentation test is shown in Figure 9a. Figure 9b is a zoomed in view which shows a very slight indentation on the surface with no other surface damages.

(a)

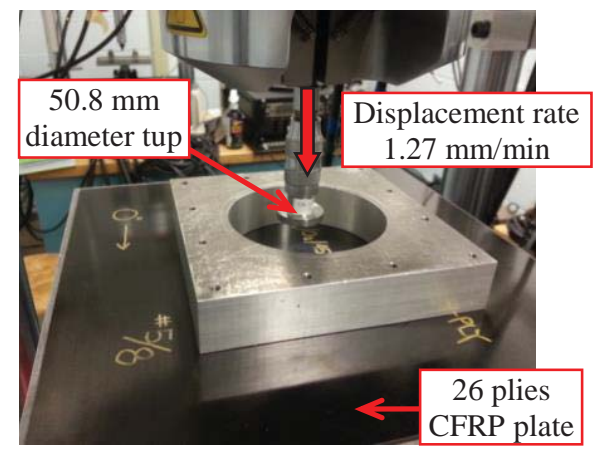

(b)

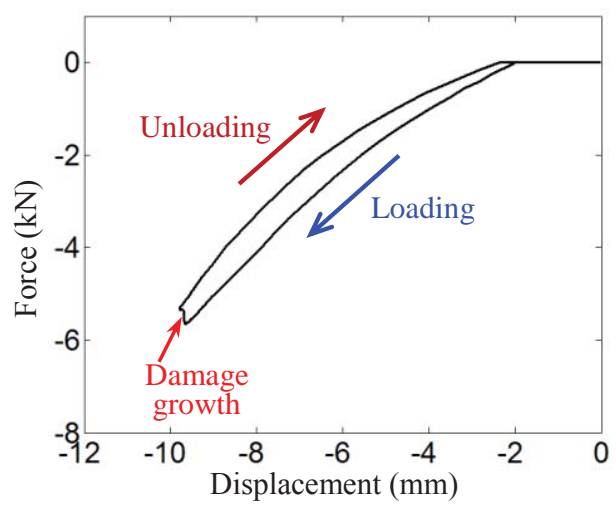

Figure 8. Quasi-static indentation for delamination damage growth: (a) the indentation setup, and (b) the curve of indentation force versus displacement.

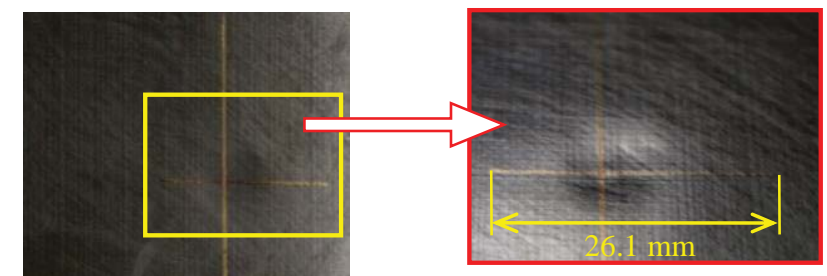

(b)

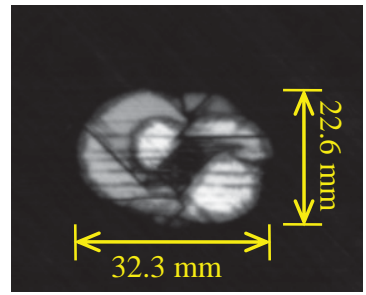

(c)

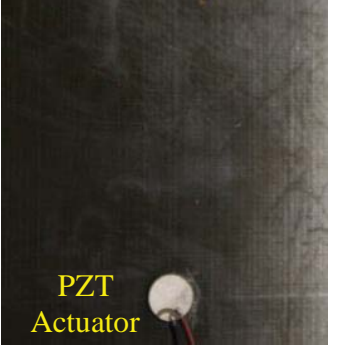

(a)

Figure 9. The 26 ply laminate after the quasi-static indentation: (a) a photo of the specimen, (b) a zoomed view of the indentation, and (c) a Cscan image showing the interior delamination damage generated by the indentation test.

Following the quasi-static indentation test, an ultrasonic Cscan was used to quantify the resulting delamination damage under the plate surface. The Cscan data were collected in an immersion tank using a $10 \mathrm{MHz}$ transducer and a scan step size of $0.25 \mathrm{~mm}$. The Cscan image in Figure 9c shows multiple delaminations within the $32.3 \mathrm{~mm} \times 22.6 \mathrm{~mm}$ damage zone, occurring at various depths through the plate thickness.

\subsection{Delamination detection and quantification}

A $7 \mathrm{~mm}$ round PZT was installed on the CFRP plate surface (shown in Figure 9a) to generate guided waves at 500 $\mathrm{kHz}$. A scanning laser Doppler vibrometer was used to measure the wavefield in the CFRP specimen containing the impactinduced delamination damage. Figure 10a is a wavefield snapshot at $55 \mu$ s showing the guided wave propagations and interactions. The incident guided waves interact with the damage and are trapped in the delamination region. Due to the existence of multiple wave modes and complex wave interactions, it is difficult to detect and quantify the delamination damage only from the original measured timedomain wavefield. Further wavefield processing is required.

To detect and quantify the damage, both the wavefield filtering and wavenumber field analysis methods were used. Figure $10 \mathrm{~b}$ gives the wavefield filtering result which only retains the trapped waves. The trapped waves clearly indicate the delamination location.

Figure 10c shows the wavenumber field over the test area. The delamination damage is clearly observed as larger wavenumbers in wavenumber field. The detected delamination size in the wavenumber field is approximately $30 \mathrm{~mm} \times 20$ $\mathrm{mm}$, which agrees well with the Cscan result. Moreover, the 
delamination shape in the wavenumber field closely matches

the delamination shape in the Cscan image Figure 9c.
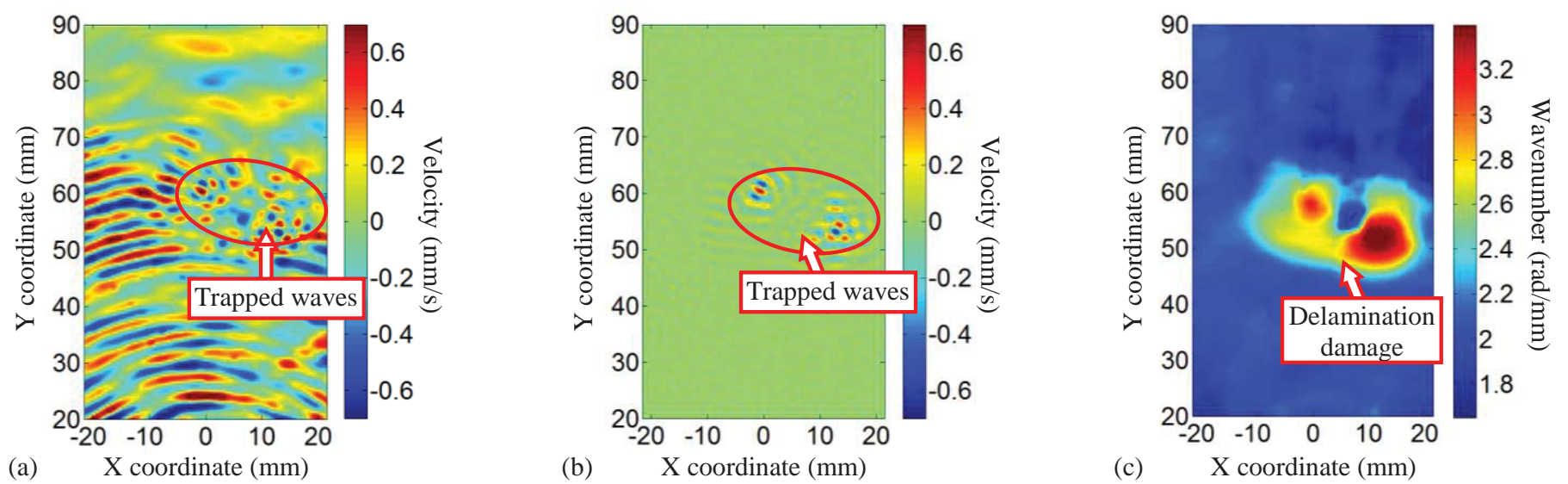

Figure 10. The delamination damage detection and quantification results: (a) the wavefield snapshot at $55 \mu s$ shows the guided wave interactions and trappings in the impact-induced delamination damage, (b) the filtered wavefield snapshot at 55 us shows the trapped waves at the damage, and (c) wavenumber field shows the location and shape of the damage.

\section{CONCLUSIONS}

This paper presents our studies on guided wave interactions with impact induced delamination damage and damage quantification techniques for laminated composite plates. The wavefield data analysis confirms the wave trapping phenomenon. Quantitative interpretation of the wave behaviors in the delamination region via different wavenumber analysis methods is demonstrated. Among the methods studied, the frequency-wavenumber analysis shows that the frequencywavenumber spectrum can indicate the presence of new wavenumbers. The reconstructed wavefield images created from the new wavenumber components demonstrate that the new wavenumbers correlate to trapped waves in the delamination region. Additionally, the wavenumber field shows how the wavenumber changes as a function of the propagation distance. Thus, the location and spatial duration of the new wavenumbers can be identified, providing a useful means not only for detecting the presence of delamination damage but also sizing the damage. Finally, the wavenumber field analysis shows the wavenumber values at each spatial point indicating not only the location and size, but also the shape of the damage.

The developed analysis methods have been applied to detect impact-induced delamination damage in a carbon fiber reinforced polymer plate. The delamination is barely visible even from careful visual inspection while C-scan images show internal delamination of about $30 \mathrm{~mm} \times 20 \mathrm{~mm}$ size. By using wavenumber field analysis, interior delamination damage is detected and quantified.

\section{Acknowledgement}

The authors acknowledge support from (1) the nonreimbursement Space Act Umbrella Agreement SAA1-1181 between South Carolina Research Foundation (SCRF) and the
National Aeronautics and Space Administration (NASA) Langley Research Center, (2) University of South Carolina SPARC graduate fellowship, and (3) SC NASA EPSCoR Research and Education Awards Program (REAP) 21-NEUSC_Yu-RGP.

\section{REFERENCES}

[1] Ip, K. H., and Mai, Y. W., 2004, "Delamination Detection in Smart Composite Beams Using Lamb Waves," Smart Materials \& Structures, 13(3), pp. 544-551.

[2] Staszewski, W. J., Mahzan, S., and Traynor, R., 2009, "Health Monitoring of Aerospace Composite Structures-Active and Passive Approach," Composites Science and Technology, 69, pp. 1678-1685.

[3] Alleyne, D. N., and Cawley, P., 1992, "The Interaction of Lamb Waves with Defects," Ieee Transactions on Ultrasonics Ferroelectrics and Frequency Control, 39, pp. 381-397.

[4] Rose, J. L., 1999, Ultrasonic Waves in Solid Media, Cambridge University Press, Cambridge.

[5] Boller, C., 2000, "Next Generation Structural Health Monitoring and Its Integration into Aircraft Design," International Journal of Systems Science, 31, pp. 1333-1349.

[6] Staszewski, W. J., Boller, C., and Tomlinson, G. R., 2004, Health Monitoring of Aerospace Structures, John Wiley \& Sons, Chichester.

[7] Giurgiutiu, V., 2008, Structural Health Monitoring with Piezoelectric Wafer Active Sensors, Academic Press, Boston, MA.

[8] Ong, W. H., and Chiu, W. K., 2012, "Redirection of Lamb Waves for Structural Health Monitoring," Smart Materials Research, 2012, p. 718686.

[9] Wang, L., and Yuan, F. G., 2007, "Group Velocity and Characteristic Wave Curves of Lamb Waves in Composites: 
Modeling and Experiments," Composites Science and Technology, 67(7-8), pp. 1370-1384.

[10] Kundu, T., Das, S., Martin, S. A., and Jata, K. V., 2008, "Locating Point of Impact in Anisotropic Fiber Reinforced Composite Plates," Ultrasonics, 48(3), pp. 193-201.

[11] Su, Z. Q., Cheng, L., Wang, X. M., Yu, L., and Zhou, C., 2009, "Predicting Delamination of Composite Laminates Using an Imaging Approach," Smart Materials \& Structures, 18, p. 074002 .

[12] Castaings, M., and Hosten, B., 2008, "Ultrasonic Guided Waves for Health Monitoring of High-Pressure Composite Tanks," Ndt \& E International, 41(8), pp. 648-655.

[13] Salamone, S., Bartoli, I., di Scalea, F. L., and Coccia, S., 2009, "Guided-Wave Health Monitoring of Aircraft Composite Panels under Changing Temperature," Journal of Intelligent Material Systems and Structures, 20(9), pp. 1079-1090.

[14] Salas, K. I., and Cesnik, C. E. S., 2010, "Guided Wave Structural Health Monitoring Using Clover Transducers in Composite Materials," Smart Materials \& Structures, 19, p. 015014

[15] Song, F., Huang, G. L., and Hu, G. K., 2012, "Online Guided Wave-Based Debonding Detection in Honeycomb Sandwich Structures," Aiaa Journal, 50(2), pp. 284-293.

[16] Giurgiutiu, V., and Soutis, C., 2012, "Enhanced Composites Integrity through Structural Health Monitoring," Applied Composite Materials, 19, pp. 813-829.

[17] Leckey, C. A. C., Rogge, M. D., and Parker, R., 2014, "Guided Waves in Anisotropic and Quasi-Isotropic Aerospace Composites: Three-Dimensional Simulation and Experiment," Ultrasonics, 54, pp. 385-394.

[18] Guo, N., and Cawley, P., 1993, "The Interaction of Lamb Waves with Delaminations in Composite Laminates," Journal of Acoustic Society of America, 94, pp. 2240-2246.

[19] Petculescu, G., Krishnaswamy, S., and Achenbach, J. D., 2008, "Group Delay Measurements Using Modally Selective Lamb Wave Transducers for Detection and Sizing of Delaminations in Composites," Smart Materials \& Structures, 17, p. 015007.

[20] Purekar, A. S., and Pines, D. J., 2010, "Damage Detection in Thin Composite Laminates Using Piezoelectric Phased Sensor Arrays and Guided Lamb Wave Interrogation," Journal of Intelligent Material Systems and Structures, 21(10), pp. 9951010.

[21] Michaels, T. E., Michaels, J. E., and Ruzzene, M., 2011, "Frequency-Wavenumber Domain Analysis of Guided Wavefields," Ultrasonics, 51, pp. 452-466.

[22] Yeum, C. M., Sohn, H., Ihn, J. B., and Lim, H. J., 2012, "Instantaneous Delamination Detection in a Composite Plate Using a Dual Piezoelectric Transducer Network," Composite Structures, 94, pp. 3490-3499.

[23] Rogge, M. D., and leckey, C. A. C., 2013, "Characterization of Impact Damage in Composite Laminates Using Guided Wavefield Imaging and Local Wavenumber Domain Analysis," Ultrasonics, 53(7), pp. 1217-1226.
[24] Hayashi, T., and Kawashima, K., 2002, "Multiple Reflections of Lamb Waves at a Delamination," Ultrasonics, 40, pp. 193-197.

[25] Ramadas, C., Balasubramaniam, K., Joshi, M., and Krishnamurthy, C. V., 2009, "Interaction of the Primary AntiSymmetric Lamb Mode (A0) with Symmetric Delaminations: Numerical and Experimental Studies," Smart Materials \& Structures, 18, p. 085011.

[26] Sohn, H., Dutta, D., Yang, J. Y., Park, H. J., DeSimio, M. P., Olson, S. E., and Swenson, E. D., 2011, "Delamination Detection in Composites through Guided Wave Field Image Processing," Composites Science and Technology, 71, pp. 1250-1256.

[27] Sohn, H., Dutta, D., Yang, J. Y., DeSimio, M., Olson, S., and Swenson, E., 2011, "Automated Detection of Delamination and Disbond from Wavefield Images Obtained Using a Scanning Laser Vibrometer," Smart Materials \& Structures, 20, p. 045017.

[28] Glushkov, E., Glushakova, N., Golub, M. V., Moll, J., and Fritzen, C. P., 2012, "Wave Energy Trapping and Localization in a Plate with a Delamination," Smart Materials \& Structures, 21, p. 125001.

[29] Tian, Z., Yu, L., and Leckey, C. A. C., "Guided Wave Propagation Study on Laminated Composites by FrequencyWavenumber Technique," Proc. Nondestructive Characterization for Composite Materials, Aerospace Engineering, Civil Infrastructure, and Homeland Security 2014, SPIE, p. 906310.

[30] Alleyne, D. N., and Cawley, P., 1991, "A Two Dimensional Fourier Transform Method for the Measurement of Propagating Multimode Signals," Journal of Acoustic Society of America, 89(3), pp. 1159-1168.

[31] Ruzzene, M., 2007, "Frequency-Wavenumber Domain Filtering for Improved Damage Visualization," Smart Materials and Structures, 16, pp. 2116-2129.

[32] Yu, L., and Tian, Z., 2013, "Lamb Wave Structural Health Monitoring Using a Hybrid Pzt-Laser Vibrometer Approach," Structural Health Monitoring, 12, pp. 469-483.

[33] Tian, Z., and Yu, L., 2014, "Lamb Wave FrequencyWavenumber Analysis and Decomposition," Journal of Intelligent Material Systems and Structures, p. in press.

[34] Johnson, D. H., and Dudgeon, D. E., 1993, Array Signal Processing: Concepts and Techniques, Prentice-Hall Inc., Upper Saddle River, New Jersey, USA.

[35] Cohen, L., 1994, Time Frequency Analysis: Theory and Applications, Prentice-Hall Inc., Upper Saddle River, New Jersey, USA.

[36] Williams, G., Trask, R., and Bond, I., 2007, "A SelfHealing Carbon Fibre Reinforced Polymer for Aerospace Applications," Composites Part a-Applied Science and Manufacturing, 38(6), pp. 1525-1632. 